\title{
Topological black holes in the dimensionally continued gravity
}

\author{
Rong-Gen Cai \\ Center for Theoretical Physics, Seoul National University, Seoul 151-742, Korea \\ Kwang-Sup Soh \\ Department of Physics Education, Seoul National University, Seoul 151-742, Korea
}

\begin{abstract}
We investigate the topological black holes in a special class of Lovelock gravity. In the odd dimensions, the action is the Chern-Simons form for the anti-de Sitter group. In the even dimensions, it is the Euler density constructed with the Lorentz part of the anti-de Sitter curvature tensor. The Lovelock coefficients are reduced to two independent parameters: cosmological constant and gravitational constant. The event horizons of these topological black holes may have constant positive, zero or negative curvature. Their thermodynamics is analyzed and electrically charged topological black holes are also considered. We emphasize the differences due to the different curvatures of event horizons. As a comparison, we also discuss the topological black holes in the higher dimensional Einstein-Maxwell theory with a negative cosmological constant.
\end{abstract}

PACS numbers: 04.20.Jb, 04.20.Gz, 97.60.Lf 


\section{INSTRUCTION}

Over the past few years there has been a lot of interest in black holes in the anti-de Sitter spacetimes. This study is motivated by the discovery of Bañados-Teitelboim-Zanelli (BTZ) black holes [1], which are exact solutions in the three-dimensional Einstein gravity with a negative cosmological constant, and are locally equivalent to a three-dimensional anti-de Sitter space. That is, the BTZ black holes can be constructed by identifying some discrete points along a boost Killing vector in the three-dimensional anti-de Sitter space [2]. Using such kind of identifications, the so-called constant curvature black holes can also be constructed in the four-dimensional [3] as well as higher dimensional [⿴囗十 anti-de Sitter spacetimes. The Euclidean manifold topologies of these black holes are $R^{D-1} \times S^{1}$, where $S^{1}$ is the topology of event horizons, in contrast to the usual topology of black holes $R^{2} \times S^{D-2}$. Because of the unusual asymptotic behavior of these constant curvature black holes, however, identifying the globally conserved quantities seems difficult (For a quasilocal formulation see [5]).

On the other hand, except for the Kerr-Newmann-anti-de Sitter black hole, whose event horizon has the topology $S^{2}$, in the four-dimensional Einstein-Maxwell theory with a negative cosmological constant, it has been found recently that there exist black hole solutions whose event horizons may have zero or negative constant curvature and their topologies are no longer the two-sphere $S^{2}$. Because of the different topological structures of even horizons, properties of these black holes are quite different from those of black holes with usual spherical topology horizon. These black holes have been studied extensively in many aspects such as exact solutions [6-10], thermodynamics [11, 12], pair production [13], gravitational collapse [14, 15], and others [16, 21].

So far, most of the works have been limited in the Einstein gravitational theory. Quite recently, Klemm [10] has found topological black hole solutions in the Weyl conformal gravity. In a previous paper, we have investigated the topological black holes [22] in a class of dilaton gravity with a Liouville-type dilaton potential. Differing from the topological black holes in the Einstein-Maxwell theory, which approach asymptotically the anti-de Sitter spaces, the topological dilaton black holes are asymptotically neither the anti-de Sitter spaces nor de Sitter spaces or Minkowski spacetimes. But the negative effective cosmological constant plays a crucial role in the existence of these black hole solutions, as the negative cosmological constant does in the Einstein-Maxwell theory.

In the present paper, we would like to investigate the topological black holes in the higher dimensional spacetimes. In the Einstein-Maxwell theory, the higher dimensional, spherically symmetric black holes have been studied by Myers and Perry [23. And their analogues in the Brans-Dicke theory have been investigated recently in [24]. Therefore, four-dimensional topological black holes have their natural generalization in the higher dimensional EinsteinMaxwell theory with a negative cosmological constant. For example, there are the static topological black holes in four-dimensional spacetimes

$$
d s^{2}=-\left(k-\frac{8 \pi M}{\omega_{2} r}+\frac{16 \pi^{2} Q^{2}}{\omega_{2}^{2} r^{2}}+\frac{r^{2}}{l^{2}}\right) d t^{2}+\left(k-\frac{8 \pi M}{\omega_{2} r}+\frac{16 \pi^{2} Q^{2}}{\omega_{2}^{2} r^{2}}+\frac{r^{2}}{l^{2}}\right)^{-1} d r^{2}+r^{2} d \Sigma_{2}^{2},
$$

where $d \Sigma_{2}^{2}$ is the line element of a two-dimensional hypersurface $\Sigma_{2}$ with constant curvature 
$2 k$,

$$
d \Sigma_{2}^{2}= \begin{cases}d \theta^{2}+\sin ^{2} \theta d \phi^{2} & \text { for } \quad k=1, \\ d \theta^{2}+\theta^{2} d \phi^{2} & \text { for } \quad k=0, \\ d \theta^{2}+\sinh ^{2} \theta d \phi^{2} & \text { for } \quad k=-1\end{cases}
$$

Here $M$ and $Q$ are the mass and charge of the black holes, $-3 l^{-2}$ is the negative cosmological constant, and $\omega_{2}$ is the area of the horizon hypersurface $\Sigma_{2}$. In (1.2), without loss of the generality, we have used the coordinates in which the constant curvature of the twodimensional hypersurface of the event horizon is 1,0 , and -1 , respectively. When $k=1$, the solution (1.1) is just the Reissner-Nordström-anti-de Sitter black hole spacetime and the event horizon has the topology $S^{2}$. When $k=0$, if one identities the coordinates $\theta$ and $\phi$ with certain periods, the resulting topology of event horizon is a torus $T^{2}$. The event horizon is a hyperbolic surface as $k=-1$. Of interest is to note that the event horizon still appears even if the mass $M$ is negative in that case, and such kind of negative mass black holes might be formed by the regular gravitational collapse [14]. In addition, because of the different topological structures of event horizons, their thermodynamic behaviors are quite different [11.12]. As a natural extension, we have topological black hole solutions in the higher dimensional Einstein-Maxwell theory with a negative cosmological constant $\Lambda=-(D-1)(D-2) / 2 l^{2}$ :

$$
\begin{aligned}
d s^{2} & =-\left(k-\frac{16 \pi M}{(D-2) \omega_{D-2} r^{D-3}}+\frac{16 \pi^{2} Q^{2}}{\omega_{D-2}^{2} r^{2(D-3)}}+\frac{r^{2}}{l^{2}}\right) d t^{2} \\
& +\left(k-\frac{16 \pi M}{(D-2) \omega_{D-2} r^{D-3}}+\frac{16 \pi^{2} Q^{2}}{\omega_{D-2}^{2} r^{2(D-3)}}+\frac{r^{2}}{l^{2}}\right)^{-1} d r^{2}+r^{2} d \Sigma_{D-2}^{2},
\end{aligned}
$$

where $d \Sigma_{D-2}^{2}=\gamma_{m n} d x^{m} d x^{n}$ is a (D-2)-dimensional hypersurface $\Sigma_{D-2}$ with constant curvature $(D-2) k$, and $\omega_{D-2}$ is its area. Without loss of generality, one may normalize the constant curvature to $k=1,0$, and -1 , respectively. These black holes have similar properties as those in the four-dimensional spacetime. For the discussion about the higher dimensional topological uncharged black holes see [25].

Instead of the pure Einstein gravity, in this paper, we consider the topological black holes in the so-called dimensionally continued gravity [26]. This theory will be reviewed briefly in the next section. The topological black hole solutions will be presented and discussed in Sec. III. The section IV is devoted to the case including the Maxwell field. We summarize our results in Sec.V. In the Appendix we will discuss the thermodynamics of the topological black holes (1.3) in the Einstein-Maxwell theory.

\section{DIMENSIONALLY CONTINUED GRAVITY}

The dimensionally continued gravity is a special class of the Lovelock gravity [27], which may be regarded as the most general generalization to higher dimensions of the Einstein gravity. The Lovelock action is a sum of the dimensionally continued Euler characteristics of all dimensions below the spacetime dimension $D(\geq 3)$ under consideration. It can be written down as [26] 


$$
I=\kappa \sum_{p=0}^{n} \alpha_{p} I_{p}
$$

where

$$
I_{p}=\int \epsilon_{a_{1} \cdots a_{D}} R^{a_{1} a_{2}} \wedge \cdots \wedge R^{a_{2 p-1} a_{2 p}} \wedge e^{a_{2 p+1}} \wedge \cdots \wedge e^{a_{D}} .
$$

Here $e^{a}$ is the local frame one-form, $R_{b}^{a}$ is the curvature two-form defined as $R_{b}^{a}=d w_{b}^{a}+$ $w^{a}{ }_{c} \wedge w^{c}{ }_{b}$, and $w_{b}^{a}$ is the spin connection, $a_{i}=\{0,1, \cdots, D-1\}$. The coefficients $\alpha_{p}$ are arbitrary constants with dimensions [length $]^{-(D-2 p)}$ and $\kappa$ has units of action.

The Lovelock action (2.1) has the advantage which keeps the field equations of motion for the metric of second order, as the pure Einstein-Hilbert action. But it includes $[D / 2]$ arbitrary constants $\alpha_{p}$, which makes difficult to extract physical information from the solutions of the equations of motions. In [26] a proposal has been suggested to reduce these arbitrary constants to two: a cosmological constant and a gravitational constant. This proposal was made by embedding the Lorentz group $S O(D-1,1)$ into a larger group, anti-de Sitter group $S O(D-1,2)$. In this way the Lovelock theory is divided into two different branches according to the spacetime dimensions: odd dimensions and even dimensions. The coefficients $\alpha_{p}$ are given by

$$
\alpha_{p}= \begin{cases}\frac{1}{D-2 p}\left(\begin{array}{c}
n-1 \\
p
\end{array}\right) l^{-D+2 p} & \text { for } D=2 n-1 \\
\left(\begin{array}{c}
n \\
p
\end{array}\right) l^{-D+2 p} & \text { for } D=2 n\end{cases}
$$

where $l$ is a length.

In the odd dimensions, the Lagrangian $\mathcal{L}_{2 n-1}$ is

$$
\mathcal{L}_{2 n-1}=\kappa \sum_{0}^{n-1} \alpha_{p} \epsilon_{a_{1} \cdots a_{D}} R^{a_{1} a_{2}} \wedge \cdots \wedge R^{a_{2 p-1} a_{2 p}} \wedge e^{a_{2 p+1}} \wedge \cdots \wedge e^{a_{D}} .
$$

For later convenience, the units are chosen so that

$$
\kappa=\frac{l}{(D-2) ! \omega_{D-2}} \quad \text { for } D=2 n-1
$$

where $\omega_{D-2}$ is the area of a $(D-2)$-dimensional hypersurface $\Sigma_{D-2}$ which will be defined later. In the even dimensions, the Lagrangian $\mathcal{L}_{2 n}$ is given by

$$
\mathcal{L}_{2 n}=\kappa\left(R^{a_{1} a_{2}}+l^{-2} e^{a_{1}} \wedge e^{a_{2}}\right) \wedge \cdots \wedge\left(R^{a_{D-1} a_{D}}+l^{-2} e^{a_{D-1}} \wedge e^{a_{D}}\right) \epsilon_{a_{1} a_{2} \cdots a_{D}}
$$

where we choose the units so that

$$
\kappa=\frac{l^{2}}{2 D(D-2) ! \omega_{D-2}} \quad \text { for } D=2 n .
$$

In the cases $D=4$ and $D=3$, the two Lagrangians reduce to that of the Einstein gravity with a negative cosmological constant. For the details of the construction of the two Lagrangians see [26]. 
Correspondingly, the equations of motion from (2.4) and (2.6) can be derived as

$$
\left(R^{a_{1} a_{2}}+l^{-2} e^{a_{1}} \wedge e^{a_{2}}\right) \wedge \cdots \wedge\left(R^{a_{2 n-3} a_{2 n-2}}+l^{-2} e^{2 n-3} \wedge e^{2 n-2}\right) \epsilon_{a_{1} a_{2} \cdots a_{2 n-1}}=0
$$

in the odd dimensions $(D=2 n-1)$, and

$$
\left(R^{a_{1} a_{2}}+l^{-2} e^{a_{1}} \wedge e^{a_{2}}\right) \wedge \cdots \wedge\left(R^{a_{2 n-3} a_{2 n-2}}+l^{-2} e^{2 n-3} \wedge e^{2 n-2}\right) \wedge e^{2 n-1} \epsilon_{a_{1} a_{2} \cdots a_{2 n}}=0
$$

in the even dimensions $(D=2 n)$, from which it is easy to see that the anti-de Sitter space is a special solution to these equations of motion.

In [26], the static, spherically symmetric black hole solutions are obtained. The metric of the black hole solutions is

$$
d s^{2}=-g^{2}(r) d t^{2}+g^{-2}(r) d r^{2}+r^{2} d \Omega^{2},
$$

where

$$
g^{2}= \begin{cases}1-(2 M / r)^{\frac{1}{n-1}}+(r / l)^{2} & \text { for } \quad D=2 n \\ 1-(M+1)^{\frac{1}{n-1}}+(r / l)^{2} & \text { for } \quad D=2 n-1,\end{cases}
$$

$M$ is the mass of the hole and $d \Omega^{2}$ is the metric on the unit $(D-2)$-sphere. Although this black hole solution (2.10) has different quantum properties from the higher dimensional Schwarzschild-anti-de Sitter black hole, its Euclidean topology is still $R^{2} \times S^{D-2}$, where $S^{D-2}$ is the topology of its event horizon. That is, its event horizon is a $(D-2)$-dimensional sphere. In this work, we pay attention to the black holes whose event horizons are $(D-2)$ dimensional hypersurfaces with constant curvature which may be positive, zero or negative, and hence the topology of event horizon is no longer the $(D-2)$-dimensional sphere $S^{D-2}$. Here we should mention that, in this dimensionally continued gravity considered above, the Oppenheimer-Snyder gravitational collapse in the case of even dimensions has been studied recently by Ilha and Lemos [28], it has been found that the even dimensional black holes (2.10) emerge as the final state of regular dust fluid. The wormhole solutions have been found in [29].

\section{TOPOLOGICAL BLACK HOLES AND THERMODYNAMICS}

In this section we discuss topological black hole solutions to the equations of motion (2.8) and (2.9). The event horizon of these topological black holes is a $(D-2)$-dimensional hypersurface with constant curvature. The topology of horizon may be sphere, torus or other higher genus Riemann surfaces.

\section{A. Static solutions and general consideration of thermodynamics}

In order to obtain simplified equations of motion, it turns out that it is more convenient to work in the Hamiltonian form [26]. The Hamiltonian formulation of the Lovelock action (2.1) has been provided in [37]. The Hamiltonian constraint is 


$$
\mathcal{H}=-\sqrt{\operatorname{det}\left(h_{i j}\right)} \sum_{p=0}^{n-1} \frac{D-2 p}{2^{p}} \alpha_{p} \delta_{\left[j_{1} \cdots j_{2 p}\right]}^{\left[i_{1} \cdots i_{2 p}\right]} \tilde{R}_{i_{1} i_{2}}^{j_{1} j_{2}} \tilde{R}_{i_{3} i_{4}}^{j_{3} j_{4}} \cdots \tilde{R}_{i_{2 p-1} i_{2 p}}^{j_{2 p-1} j_{2 p}},
$$

where $\tilde{R}_{k l}^{i j}$ are the spatial components of the Riemann tensor. They depend on the velocities through the Gauss-Codazzi equations

$$
\tilde{R}_{i j k l}=R_{i j k l}+K_{i k} K_{j l}-K_{i l} K_{j k},
$$

where $R_{i j k l}$ are the components of the intrinsic curvature tensor of the spatial sections and $K_{i j}$ is the second fundamental form defined as $K_{i j}=\frac{1}{2 N^{\perp}}\left(-\dot{h}_{i j}+N_{i ; j}+N_{j ; i}\right)$, where $h_{i j}, N^{\perp}$, and $N^{i}$ are the reduced metric, lapse function and shift vectors in the standard ArnowittDeser-Misner (ADM) decomposition of spacetime.

We are looking for the static topological black hole solutions. So the metric are assumed as

$$
d s^{2}=-N^{2}(r) g^{2}(r) d t^{2}+g^{-2}(r) d r^{2}+r^{2} d \Sigma_{D-2}^{2},
$$

where $d \Sigma_{D-2}^{2}=\gamma_{m n}(x) d x^{m} d x^{n}$ is the metric of $(D-2)$-dimensional hypersurface $\Sigma_{D-2}$ with constant curvature $(D-2) k$ and its area is denoted by $\omega_{D-2}$, which is just the one in (2.5) and (2.7). The functions $N^{2}(r)$ and $g^{2}(r)$ need to be determined. In the metric (3.3) the nonvanishing spatial components of curvature tensors are

$$
\begin{aligned}
& R_{n_{1} n_{2}}^{m_{1} m_{2}}=\frac{f(r)}{r^{2}} \delta_{\left[n_{1} n_{2}\right]}^{\left[m_{1} m_{2}\right]} \\
& R_{r n}^{r m}=\frac{f^{\prime}(r)}{2 r} \delta_{n}^{m}
\end{aligned}
$$

where a prime denotes the derivative with respect to $r$ and $f(r)=k-g^{2}(r)$. Substituting (3.4) into the Hamiltonian constraint (3.1) yields

$$
\mathcal{H}=-(D-2) ! \sqrt{\gamma} g^{-1}\left[r^{D-1} \sum_{p=0}^{n-1} \alpha_{p}(D-2 p)\left(\frac{k-g^{2}}{r^{2}}\right)^{p}\right]^{\prime}
$$

Using the coefficients (2.3) and units (2.5) and (2.7), one has the action

$$
I=\left(t_{2}-t_{1}\right) \int d r N F^{\prime}(r)+B
$$

where $B$ stands for a surface term and the function $F$ is given by

$$
F[r, g(r)]= \begin{cases}\frac{1}{2} r\left[k+(r / l)^{2}-g^{2}(r)\right]^{n-1} & \text { for } D=2 n \\ {\left[k+(r / l)^{2}-g^{2}(r)\right]^{n-1}} & \text { for } D=2 n-1 .\end{cases}
$$

Varying the action (3.6) with respect to $N$ and $F$, one has the equations of motion

$$
\frac{d F}{d r}=0, \quad \frac{d N}{d r}=0
$$

which have solutions 


$$
\begin{aligned}
& F[r, g(r)]=\tilde{C} \equiv \text { Const } \\
& N(r)=N_{\infty} \equiv \text { Const. }
\end{aligned}
$$

Here the integration constant $N_{\infty}$ can be taken to be one by rescaling the time coordinate $t$. And the constant $\tilde{C}$ is related to, up to an additive constant, the mass $M$ of black holes,

$$
\tilde{C}=M+\tilde{C}_{0} .
$$

This additive constant $\tilde{C}_{0}$ is achieved by choosing an appropriate reference background. Thus we obtain the metric function $g^{2}(r)$ :

$$
g^{2}(r)= \begin{cases}k-\left(\frac{2 M+2 \tilde{C}_{0}}{r}\right)^{\frac{1}{n-1}}+\left(\frac{r}{l}\right)^{2} & \text { for } \quad D=2 n \\ k-\left(M+\tilde{C}_{0}\right)^{\frac{1}{n-1}}+\left(\frac{r}{l}\right)^{2} & \text { for } \quad D=2 n-1\end{cases}
$$

The mass for the even dimensional solutions has the dimensions of length, and is dimensionless for the odd dimensional solutions. This is because we have chosen the different units (2.5) and (2.7). To analyze the singularities of the solutions (3.11), let us write down some curvature invariants

$$
\begin{aligned}
& R= \begin{cases}-\frac{D(D-1)}{l^{2}}+\left[\frac{2 D}{(D-2)^{2}}+D^{2}-5 D+2\right] \frac{1}{r^{2}}\left(\frac{2 M+2 \tilde{C}_{0}}{r}\right)^{\frac{1}{n-1}} & \text { for } D=2 n, \\
-\frac{D(D-1)}{l^{2}}+\frac{(D-2)(D-3)}{l^{2}}\left(M+\tilde{C}_{0}\right)^{\frac{1}{n-1}} & \text { for } D=2 n-1 .\end{cases} \\
& R_{\alpha \beta} R^{\alpha \beta}=\frac{1}{2}\left[\left(g^{2}\right)^{\prime \prime}\right]^{2}+\frac{(D-2)}{r}\left(g^{2}\right)^{\prime \prime}\left(g^{2}\right)^{\prime}+\frac{D(D-2)}{2 r^{2}}\left[\left(g^{2}\right)^{\prime}\right]^{2} \\
& -\frac{2(D-2)(D-3)}{r^{3}}\left(k-g^{2}\right)\left(g^{2}\right)^{\prime}+\frac{(D-2)(D-3)}{r^{4}}\left(k-g^{2}\right)^{2} \text {. } \\
& R_{\alpha \beta \gamma \delta} R^{\alpha \beta \gamma \delta}=\left[\left(g^{2}\right)^{\prime \prime}\right]^{2}+\frac{2(D-2)}{r^{2}}\left[\left(g^{2}\right)^{\prime}\right]^{2}+\frac{2(D-2)(D-3)}{r^{4}}\left(k-g^{2}\right)^{2} .
\end{aligned}
$$

Before proceeding to discuss the solution (3.11) for different curvature $k$, let us consider generally the thermodynamics of black hole solutions (3.3). Assuming the metric (3.3) describes a black hole with event horizon at $r_{+}$, one has a black hole with event horizon being a $(D-2)$-dimensional hypersurface $\Sigma_{D-2}$ whose curvature may be positive, zero or negative. Apart from the topology structure of horizons, to determine the Hawking temperature of the hole, it turns out that it is convenient to continue the metric (3.3) to its Euclidean manifold with the Euclidean time $\tau=i t$,

$$
d s^{2}=N^{2}(r) g^{2}(r) d \tau^{2}+g^{-2}(r) d r^{2}+r^{2} d \Sigma_{D-2}^{2} .
$$

For an arbitrary period of the Euclidean time $\tau$, there is a conical singularity at the black hole horizon. To remove this singularity in the Euclidean manifold (3.15), one has to take a special period whose inverse just gives the Hawking temperature of black holes

$$
T=\left.\frac{\left(N^{2} g^{2}\right)^{\prime}}{4 \pi N}\right|_{r=r_{+}} .
$$


For the solutions (3.11), Using (3.16) yields the Hawking temperature of the holes

$$
T= \begin{cases}\frac{k+(2 n-1)\left(r_{+} / l\right)^{2}}{4 \pi(n-1) r_{+}} & \text {for } D=2 n, \\ \frac{r_{+}}{2 \pi l^{2}} & \text { for } D=2 n-1 .\end{cases}
$$

Usually the black hole entropy satisfies the so-called area formula. That is, the black hole entropy equals to one quarter of event horizon area. But this formula does not always hold. It has been proved that this formula holds only for the Einstein gravity and in fact, black hole entropy comes from a surface term of gravitational action at the horizon [24, 31, 32, That is, the black hole entropy is related to the gravitational theory under consideration. To get the black hole entropy, there are several methods available now. Here we adopt a simpler method for this goal. This method is based on the fact that, as thermodynamic systems, black holes must obey the first law of thermodynamics

$$
d M=T d S+\sum_{i=1} \mu_{i} Q_{i}
$$

where $M$ is the mass of black holes, $T$ and $S$ are the Hawking temperature and HawkingBekenstein entropy of the black holes, respectively. $\mu_{i}$ are the chemical potentials corresponding to the conserved charges $Q_{i}$. Using (3.18) one has

$$
\begin{aligned}
S & =\int T^{-1} d M+S_{0} \\
& =\int T^{-1}\left(\frac{\partial M}{\partial r_{+}}\right)_{Q_{i}} d r_{+}+S_{0} .
\end{aligned}
$$

Here it should be reminded that, in the integration (3.19), the charges $Q_{i}$ should be taken as constants. $S_{0}$ is an integration constant, which can be fixed by using the argument that the black hole entropy should vanish as the event horizon of black holes disappears. Therefore, the expression (3.19) can be rewritten as

$$
S=\int_{0}^{r_{+}} T^{-1}\left(\frac{\partial M}{\partial r_{+}}\right)_{Q_{i}} d r_{+} .
$$

Thus, once given the Hawking temperature and the mass expressed by the horizon radius $r_{+}$and charges $Q_{i}$, one can obtain the entropy of the black hole and needs not to know in which gravitational theory the black hole solutions are.

For the solutions (3.11), the charges $Q_{i}$ are absent. Using the Hawking temperature (3.17), we obtain the black hole entropy of solutions (3.11)

$$
\begin{aligned}
S & =2 \pi(n-1) \int_{0}^{r_{+}} r_{+}\left[k+\left(\frac{r_{+}}{l}\right)^{2}\right]^{n-2} d r_{+} \\
& =\pi l^{2}\left\{\left[k+\left(\frac{r_{+}}{l}\right)^{2}\right]^{n-1}-k\right\},
\end{aligned}
$$

in the even dimensions, and 


$$
\begin{aligned}
S & =4 \pi(n-1) \int_{0}^{r_{+}}\left[k+\left(\frac{r_{+}}{l}\right)^{2}\right]^{n-2} d r_{+} \\
& =4 \pi(n-1) \int_{0}^{r_{+}} \sum_{m=0}^{n-2}\left(\begin{array}{c}
n-2 \\
m
\end{array}\right)\left(\frac{r_{+}}{l}\right)^{2 m} k^{n-2-m} d r_{+} \\
& =4 \pi(n-1) l \sum_{m=0}^{n-2}\left(\begin{array}{c}
n-2 \\
m
\end{array}\right) \frac{1}{2 m+1}\left(\frac{r_{+}}{l}\right)^{2 m+1} k^{n-2-m} .
\end{aligned}
$$

in the odd dimensions. Obviously, they do not obey the usual area formula.

To see the stability of black holes against the Hawking radiation, it is useful to compute the heat capacity defined as $C_{Q_{i}} \equiv(\partial M / \partial T)_{Q_{i}}$. For the solutions (3.11), we obtain

$$
C= \begin{cases}2 \pi(n-1) r_{+}^{2} \frac{\left[k+\left(r_{+} / l\right)^{2}\right]^{n-2}\left[(2 n-1)\left(r_{+} / l\right)^{2}+k\right]}{(2 n-1)\left(r_{+} / l\right)^{2}-k} & \text { for } D=2 n, \\ 4 \pi(n-1) r_{+}\left[k+\left(r_{+} / l\right)^{2}\right]^{n-2} & \text { for } D=2 n-1 .\end{cases}
$$

Here we would like to stress that these physical quantities (3.17), (3.21), (3.22) and (3.23) are all expressed in terms of the horizon radius $r_{+}$and curvature $k$, the addition constant $\tilde{C}_{0}$ does not explicitly enter these quantities.

We now turn to discussing the solutions (3.11) for different horizon curvature $k$. As mentioned in the Introduction, without loss of generality, the curvature can be normalized as $k=1,0$ and -1 , respectively. In the case $k=1$ and horizon is spherical topology, this solution has already been analyzed in some detail in [26]. Just as pointed out by Birmingham [25], however, it should be noticed that in the higher dimensions, even in the case $k=1$, there still exist the possibilities of non-spherical topology for the horizon hypersurface. In addition, to compare with the other two cases, we also summarize and comment the $k=1$ black hole solutions below.

\section{B. $k=1$ solutions}

To analyze the solutions (3.11), one has to first fix the additive constant $\tilde{C}_{0}$. In [26], Bañados, Teitelboim, and Zanelli used a criterion to fix the constant $\tilde{C}_{0}$ in (3.10) that for zero energy the horizon should disappear. They fixed the constant $\tilde{C}_{0}$ as

$$
\tilde{C}_{0}= \begin{cases}0 & \text { for } \quad D=2 n \\ 1 & \text { for } \quad D=2 n-1\end{cases}
$$

If use this choice, we have the solutions

$$
d s^{2}=-\left[1-(2 M / r)^{\frac{1}{n-1}}+(r / l)^{2}\right] d t^{2}+\left[1-(2 M / r)^{\frac{1}{n-1}}+(r / l)^{2}\right]^{-1} d r^{2}+r^{2} d \Sigma_{D-2}^{2}
$$

in the even dimensions $D=2 n$, and

$$
d s^{2}=-\left[1-(M+1)^{\frac{1}{n-1}}+(r / l)^{2}\right] d t^{2}+\left[1-(M+1)^{\frac{1}{n-1}}+(r / l)^{2}\right]^{-1} d r^{2}+r^{2} d \Sigma_{D-2}^{2},
$$

in the odd dimensions $D=2 n-1$. When $D=4$ and $D=3$, the solutions (3.25) and (3.26) reduce, respectively, to the Schwarzschild-anti-de Sitter solution and BTZ black hole solution 
[1]. For the solution (3.25), the zero mass reference background is the $D=2 n$ dimensional anti-de Sitter space. For the solution (3.26), the zero mass reference background describes in fact a zero mass black hole, the anti-de Sitter space is recovered as $M=-1$, just as happens in the BTZ black holes [1]. Here it is worth noting that, in the superstring theory, zero mass BTZ black hole and $M=-1$ anti-de Sitter space are both ground states, but in the different sectors. The anti-de Sitter space is the ground state in the NS-NS sector, while the zero mass BTZ black hole in the R-R sector [33,34].

Both of the solutions (3.25) and (3.26) approach the anti-de Sitter space, and a scalar singularity exists at $r=0$ (This singularity does not exit in the dimension $D=3$ for arbitrary mass). This singularity may be covered by an event horizon, which is determined by the equation $g^{2}(r)=0$, that is,

$$
\begin{cases}1-\left(2 M / r_{+}\right)^{\frac{1}{n-1}}+\left(r_{+} / l\right)^{2}=0 & \text { for } \quad D=2 n, \\ 1-(M+1)^{\frac{1}{n-1}}+\left(r_{+} / l\right)^{2}=0 & \text { for } \quad D=2 n-1 .\end{cases}
$$

In the even dimensions, one cannot get generally an explicit expression of horizon in terms of the mass of the hole. However, in the odd dimensions, one has

$$
r_{+}=l \sqrt{(M+1)^{\frac{1}{n-1}}-1} .
$$

In spite of the dimension, both the solutions (3.25) and (3.26) have only one horizon. In the even dimensions, the causal structure is similar to that of the Schwarzschild-anti-de Sitter black hole, while it is similar to that of the BTZ black hole in the odd dimensions. For both cases, the Penrose diagrams are exhibited in [26].

As $k=1$, the Hawking temperature (3.17) reduces to

$$
T= \begin{cases}\frac{1+(2 n-1)\left(r_{+} / l\right)^{2}}{4 \pi(n-1) r_{+}} & \text {for } D=2 n, \\ \frac{r_{+}}{2 \pi l^{2}} & \text { for } D=2 n-1 .\end{cases}
$$

Obviously, the behavior of the Hawking radiation is quite different because of the dimension. In the odd dimensions, $T \rightarrow 0$ as $r_{+} \rightarrow 0$, while $T \rightarrow \infty$ in the even dimensions. To see clearly this behavior and the stability of black holes against the Hawking radiation, let us write down the heat capacity. From (3.23), we have

$$
C= \begin{cases}2 \pi(n-1) r_{+}^{2}\left[1+\left(\frac{r_{+}}{l}\right)^{2}\right]^{n-2} \frac{\left[(2 n-1)\left(r_{+} / l\right)^{2}+1\right]}{\left[(2 n-1)\left(r_{+} / l\right)^{2}-1\right]} & \text { for } D=2 n, \\ 4 \pi(n-1) r_{+}\left[1+\left(\frac{r_{+}}{l}\right)^{2}\right]^{n-2} & \text { for } D=2 n-1 .\end{cases}
$$

From the heat capacity, we see that it is always positive in the odd dimensions. Therefore, the odd dimensional black holes can be in thermal equilibrium with Hawking radiation with arbitrary volume, as the BTZ black hole. For the even dimensional black holes, however, the heat capacity is negative as $r_{+}<l / \sqrt{2 n-1}$, positive as $r_{+}>l / \sqrt{2 n-1}$. That is, there is a transition point for the even dimensional black holes at $r_{+}=l / \sqrt{2 n-1}$, thereby the heat capacity suffers from an infinite jump.

The entropies of the wo kinds of black holes, from (3.21) and (3.22), are 


$$
S= \begin{cases}\pi l^{2}\left\{\left[1+\left(\frac{r_{+}}{l}\right)^{2}\right]^{n-1}-1\right\} & \text { for } D=2 n, \\
4 \pi(n-1) l \sum_{m=0}^{n-2}\left(\begin{array}{c}
n-2 \\
m
\end{array}\right) \frac{1}{2 m+1}\left(\frac{r_{+}}{l}\right)^{2 m+1} & \text { for } D=2 n-1 .\end{cases}
$$

As a discussion on the choice of the additive constant $\tilde{C}_{0}$ in (3.24), we note that the solution (3.26) reduces to the BTZ black hole as $D=3$ and $\tilde{C}_{0}=1$. However, for threedimensional black holes, the horizon is a circle and its curvature $k$ must vanish, i.e., $k=0$, which belongs to the class of solutions discussed in the next subsection. As a result, we have another choice of $\tilde{C}_{0}$ for the $(D>3)$ odd dimensional black holes with $k=1$. That is, one may choose $\tilde{C}_{0}=0$ as in the even dimensions, and the metric then becomes

$$
d s^{2}=-\left[1-M^{\frac{1}{n-1}}+(r / l)^{2}\right] d t^{2}+\left[1-M^{\frac{1}{n-1}}+(r / l)^{2}\right]^{-1} d r^{2}+r^{2} d \Sigma_{D-2}^{2},
$$

where $D>3$. In that case, the vacuum background, as in the even dimensions, is still the anti-de Sitter space. Note that this choice also satisfies the criterion that the horizon disappears for zero mass solutions.

\section{C. $k=0$ solutions}

In the case of $k=0$, we fix the constant $\tilde{C}_{0}$ as

$$
\tilde{C}_{0}=0, \quad \text { for arbitary dimensions. }
$$

Then we have the solutions

$$
d s^{2}=-\left[-(2 M / r)^{\frac{1}{n-1}}+(r / l)^{2}\right] d t^{2}+\left[-(2 M / r)^{\frac{1}{n-1}}+(r / l)^{2}\right]^{-1} d r^{2}+r^{2} d \Sigma_{D-2}^{2},
$$

in the even dimensions, and

$$
d s^{2}=-\left[-M^{\frac{1}{n-1}}+(r / l)^{2}\right] d t^{2}+\left[-M^{\frac{1}{n-1}}+(r / l)^{2}\right]^{-1} d r^{2}+r^{2} d \Sigma_{D-2}^{2},
$$

in the odd dimensions. When $D=3$, the solution (3.35) is the BTZ black hole solution. We refer to the ground states of these two kinds of black hole solutions as zero mass black holes, because the zero mass solutions in (3.34) and (3.35) describes a spacetime whose singularity coincides with the event horizon at $r=0$, as the zero mass BTZ solution [33]. For the solutions (3.34) and (3.35), they are both asymptotically locally equivalent to the anti-de Sitter spaces. The event horizons are

$$
r_{+}= \begin{cases}l(2 M / l)^{\frac{1}{2 n-1}} & \text { for } D=2 n \\ l M^{\frac{1}{2 n-2}} & \text { for } D=2 n-1\end{cases}
$$

Note from (3.12) that the curvature $k$ does not affect the singularity of the solution. Therefore their causal structures are similar to those of solutions for $k=1$. According to (3.17), the Hawking temperatures are 


$$
T= \begin{cases}\frac{(2 n-1) r_{+}}{4 \pi(n-1) l^{2}}=\frac{2 n-1}{4 \pi(n-1) l}(2 M / l)^{\frac{1}{2 n-1}} & \text { for } D=2 n \\ \frac{r_{+}}{2 \pi l^{2}}=\frac{1}{2 \pi l} M^{\frac{1}{2 n-2}} & \text { for } D=2 n-1 .\end{cases}
$$

Both of the Hawking temperatures approach zero as the mass goes to zero. Therefore the heat capacity should be positive. Indeed, from (3.23), one has

$$
C= \begin{cases}2 \pi(n-1) r_{+}^{2}\left(r_{+} / l\right)^{2 n-4} & \text { for } D=2 n \\ 4 \pi(n-1) r_{+}\left(r_{+} / l\right)^{2 n-4} & \text { for } \quad D=2 n-1\end{cases}
$$

which are always positive. For the even dimensional black holes, from (3.21), the entropy is

$$
S=\pi l^{2}\left(r_{+} / l\right)^{2 n-2}
$$

When $k=0$, only does the term $m=n-2$ have the contribution to the entropy in (3.22). The entropy of the odd dimensional black hole therefore is

$$
S=\frac{4 \pi(n-1) l}{2 n-3}\left(\frac{r_{+}}{l}\right)^{2 n-3} .
$$

\section{D. $k=-1$ solutions}

In the case of $k=-1$, we also fix the constant $\tilde{C}_{0}=0$ for both solutions. The black hole solutions then are

$d s^{2}=-\left[-1-(2 M / r)^{\frac{1}{n-1}}+(r / l)^{2}\right] d t^{2}+\left[-1-(2 M / r)^{\frac{1}{n-1}}+(r / l)^{2}\right]^{-1} d r^{2}+r^{2} d \Sigma_{D-2}^{2}$

in the even dimensions, and

$$
d s^{2}=-\left[-1-M^{\frac{1}{n-1}}+(r / l)^{2}\right] d t^{2}+\left[-1-M^{\frac{1}{n-1}}+(r / l)^{2}\right]^{-1} d r^{2}+r^{2} d \Sigma_{D-2}^{2},
$$

in the odd dimensions $(D>3)$. Both of the two solutions asymptotically approach the anti-de Sitter spaces. For both cases, the zero mass solution is

$$
d s^{2}=-\left[-1+(r / l)^{2}\right] d t^{2}+\left[-1+(r / l)^{2}\right]^{-1} d r^{2}+r^{2} d \Sigma_{D-2}^{2}
$$

from which one can see clearly that the zero mass solution is a black hole solution with horizon at $r_{+}=l$. Using (3.16), the Hawking temperature of the solution is found to be

$$
T=\frac{1}{2 \pi l}
$$

With the help of (3.21) and (3.22), the entropy of the zero mass black holes is

$$
S= \begin{cases}\pi l^{2} & \text { for } D=2 n \\
4 \pi(n-1) l \sum_{m=0}^{n-2}\left(\begin{array}{c}
n-2 \\
m
\end{array}\right) \frac{(-1)^{n-2-m}}{2 m+1} & \text { for } D=2 n-1\end{cases}
$$

From the above, one can find that, for the same class of solutions (3.43), when embedded in different gravities, the same black hole solution has the same Hawking temperature, but 
different entropy formula. but, this is not surprising. This is because black hole entropies are related to the gravitational theories under consideration and come from a surface term of gravitational action. So they are different for different gravitational theories. An explicit example is that the entropy of the BTZ black hole is proportional to the length of outer horizon $r_{+}$in the Einstein theory, but to the length of inner horizon in the topological gravity [35. Note that the zero mass solution (3.43) is also a special solution in the Einstein-Maxwell theory (1.3). We will show in the Appendix that the entropy of black holes (1.3) obey the area formula. That is, the entropy of zero mass black hole in (1.3) is also different from the one in (3.45).

In the general case, i.e., $M \neq 0$, we cannot get an explicit expression of the horizon in terms of the mass for the even dimensional black holes, but

$$
r_{+}=l \sqrt{1+M^{\frac{1}{n-1}}}
$$

for the odd dimensional black holes. According to (3.17), the Hawking temperatures are

$$
T= \begin{cases}\frac{(2 n-1)\left(r_{+} / l\right)^{2}-1}{4 \pi(n-1) l^{2}} & \text { for } D=2 n \\ \frac{r_{+}}{2 \pi l^{2}}=\frac{1}{2 \pi l} \sqrt{1+M^{\frac{1}{n-1}}} & \text { for } D=2 n-1 .\end{cases}
$$

From (3.23), one has the heat capacity

$$
C= \begin{cases}2 \pi(n-1) r_{+}^{2}\left[\left(\frac{r_{+}}{l}\right)^{2}-1\right]^{n-2} \frac{(2 n-1)\left(r_{+} / l\right)^{2}-1}{(2 n-1)\left(r_{+} / l\right)^{2}+1} & \text { for } D=2 n \\ 4 \pi(n-1) r_{+}\left[\left(r_{+} / l\right)^{2}-1\right]^{n-2} & \text { for } D=2 n-1\end{cases}
$$

Note from (3.41) that $r_{+} \geq l$, the heat capacity (3.48) is therefore always positive. The entropies of the black holes are

$$
S= \begin{cases}\pi l^{2}\left[\left(\frac{r_{+}}{l}\right)^{2}-1\right]^{n-1}+\pi l^{2} & \text { for } D=2 n \\
4 \pi(n-1) l \sum_{m=0}^{n-2}\left(\begin{array}{c}
n-2 \\
m
\end{array}\right) \frac{(-1)^{n-2-m}}{2 m+1}\left(\frac{r_{+}}{l}\right)^{2 m+1} & \text { for } D=2 n-1\end{cases}
$$

For the $k=-1$ solutions, there also exist the so-called negative mass black holes when $n=2 \tilde{k}+2(\tilde{k} \in \mathcal{Z})$. But, there is a critical value, beyond which the singularity at $r=0$ will be naked. The critical mass is

$$
M_{\mathrm{c}}=-\frac{l}{2 \sqrt{2 \tilde{k}+2}}\left[\frac{2 \tilde{k}+1}{2 \tilde{k}+2}\right]^{2 \tilde{k}+1},
$$

for the solution (3.41), and

$$
M_{\mathrm{c}}=-1
$$

for the solution (3.42). Inspecting (3.48), it is easy to see that the heat capacity is still positive for these negative mass black holes. 


\section{CHARGED TOPOLOGICAL BLACK HOLES}

Similar to the static, spherically symmetric black holes [26], the electric charge can also be incorporated to the topological black holes discussed in the previous section. The Hamiltonian action of the Maxwell field in a curved spacetime is

$$
I_{\mathrm{em}}=\int d t \int d^{D-1} x\left[p^{i} \dot{A}_{i}-\frac{1}{2} N^{\perp}\left(\beta h^{-1 / 2} p^{i} p_{i}+\frac{h^{1 / 2}}{2 \beta} F^{i j} F_{i j}\right)+\varphi p_{, i}^{i}\right]+B_{\mathrm{em}},
$$

where $N^{\perp}$ is the lapse function, $h$ is the determinant of the induced metric of the ADM decomposition of spacetime. $p^{i}$ is the momentum conjugate to the spatial components of the gauge field $A_{i}, \varphi=A_{0}$, and $B_{\mathrm{em}}$ is a surface term depending on the boundary condition. The constant $\beta$ related to chosen units may be taken conveniently to be the area of the hypersurface $\Sigma_{D-2}$. For static, electrically charged black holes in the metric (3.3), the action (4.1) can be reduced to [26]

$$
I_{\mathrm{em}}=\left(t_{2}-t_{1}\right) \int d r\left[-\frac{1}{2} N r^{D-2} p^{2}+\varphi\left(r^{D-2} p\right)^{\prime}\right]+B_{\mathrm{em}}
$$

where

$$
p=\frac{\beta p^{r}}{\sqrt{\gamma} r^{D-2}}
$$

Combining (4.2) and (3.6), one has the reduced action of the system

$$
I=\left(t_{2}-t_{1}\right) \int d r\left[N\left(F^{\prime}-\frac{1}{2} r^{D-2} p^{2}\right)+\varphi\left(r^{D-2} p\right)^{\prime}\right]+\tilde{B},
$$

where $F$ is still given by (3.7) and $\tilde{B}$ denotes another surface term. Varying the action (4.4) with respect to $N, g, p$, and $\varphi$, respectively, one has the equations of motion

$$
\begin{aligned}
& F^{\prime}=\frac{1}{2} r^{D-2} p^{2}, \\
& N^{\prime}=0 \\
& \varphi^{\prime}=-N p \\
& \left(r^{D-2} p\right)^{\prime}=0 .
\end{aligned}
$$

The solutions of the above equations are easily found

$$
\begin{aligned}
& N=N_{\infty}, \\
& p=\frac{Q}{r^{D-2}}, \\
& \varphi=\frac{N_{\infty} Q}{(D-3) r^{D-3}}+\varphi_{\infty}, \\
& F=-\frac{Q^{2}}{2(D-3) r^{D-3}}+\tilde{C} .
\end{aligned}
$$


Here $\tilde{C}=M+\tilde{C}_{0}, \varphi_{\infty}$ is the value of $\varphi$ at the infinity, which is conjugate to the electric charge $Q$ of the solution, and the integration constant $N_{\infty}$ is the value of $N$ at the infinity which is conjugate to the mass $M$. Therefore one can take $N_{\infty}=1$ by adjusting the time coordinate. In that case, we have solutions

$$
d s^{2}=-g^{2}(r) d t^{2}+g^{-2}(r) d r^{2}+r^{2} d \Sigma_{D-2}^{2},
$$

where

$$
g^{2}(r)= \begin{cases}k+\left(\frac{r}{l}\right)^{2}-\left[\frac{2 M+2 \tilde{C}_{0}}{r}-\frac{Q^{2}}{(D-3) r^{D-2}}\right]^{\frac{1}{n-1}} & \text { for } D=2 n \\ k+\left(\frac{r}{l}\right)^{2}-\left[M+\tilde{C}_{0}-\frac{Q^{2}}{2(D-3) r^{D-3}}\right]^{\frac{1}{n-1}} & \text { for } \quad D=2 n-1\end{cases}
$$

Once again, the additive constant $\tilde{C}_{0}$ determines the ground states of the solutions and can be fixed as in the previous section. When $D=3, \varphi$ and $F$ in (4.11) and (4.12) should be replaced by

$$
\begin{aligned}
& \varphi=-N_{\infty} Q \ln r+\varphi_{0}, \\
& F=\frac{1}{2} Q^{2} \ln r+\tilde{C}
\end{aligned}
$$

where $\varphi_{o}$ is an integration constant which is related to the choice of zero electric potential. The metric function $g^{2}$ becomes

$$
g^{2}(r)=-M+(r / l)^{2}-\frac{1}{2} Q^{2} \ln r
$$

which is just the charged BTZ black hole solution [1].

We are not going to separately analyze here the solutions (4.14) for different curvature $k$. Instead we will give a unified description. Just as the case of charged, spherically symmetric solutions [26], our solutions (4.14) may have two, one or no horizons depending on the relative value of the mass and charge. Therefore the causal structure is similar to that of the Reissner-Nordström-anti-de Sitter black hole. But, we should notice that except for the singularity at $r=0$, the solutions (4.14) have another singularity at $r=r_{c}>0$ [26] hidden by the black hole horizons $r_{-}$and $r_{+}: 0<r_{c}<r_{-}<r_{+}$, which can be see from the following curvature scalar of the solutions

$$
R=-\left(g^{2}\right)^{\prime \prime}-\frac{2(D-2)}{r}\left(g^{2}\right)^{\prime}+\frac{(D-2)(D-3)}{r^{2}}\left(k-g^{2}\right) .
$$

In general, it is difficult to get an explicit expression of horizon of black hole solutions (4.14) in terms of the mass and charge. However, we can obtain an expression of mass in terms of the horizon $r_{+}$and charge. From (4.14), we have

$$
M+\tilde{C}_{0}= \begin{cases}\frac{r_{+}}{2}\left[\left(k+\left(\frac{r_{+}}{l}\right)^{2}\right)^{n-1}+\frac{Q^{2}}{(D-3) r_{+}^{D-2}}\right] & \text { for } D=2 n, \\ {\left[k+\left(\frac{r_{+}}{l}\right)^{2}\right]^{n-1}+\frac{Q^{2}}{2(D-3) r_{+}^{D-3}}} & \text { for } D=2 n-1 .\end{cases}
$$

According to the formula $(\overline{3.16})$, the Hawking temperatures are 


$$
T= \begin{cases}\frac{\left[k+\left(r_{+} / l\right)^{2}\right]^{2-n}}{2 \pi(n-1) r_{+}}\left\{\frac{1}{2}\left[k+\left(\frac{r_{+}}{l}\right)^{2}\right]^{n-2}\left[k+(2 n-1)\left(\frac{r_{+}}{l}\right)^{2}\right]-\frac{Q^{2}}{2 r_{+}^{D-2}}\right\} & \text { for } D=2 n, \\ \frac{\left[k+\left(r_{+} / l\right)^{2}\right]^{2-n}}{4 \pi(n-1)}\left\{\frac{2(n-1) r_{+}}{l^{2}}\left[k+\left(\frac{r_{+}}{l}\right)^{2}\right]^{n-2}-\frac{Q^{2}}{2 r_{+}^{D-2}}\right\} & \text { for } D=2 n-1 .\end{cases}
$$

Using (4.19), one has

$$
\left(\frac{\partial M}{\partial r_{+}}\right)_{Q}= \begin{cases}\frac{1}{2}\left[k+\left(\frac{r_{+}}{l}\right)^{2}\right]^{n-2}\left[k+(2 n-1)\left(\frac{r_{+}}{l}\right)^{2}\right]-\frac{Q^{2}}{2 r_{+}^{D-2}} & \text { for } D=2 n \\ \frac{2(n-1) r_{+}}{l^{2}}\left[k+\left(\frac{r_{+}}{l}\right)^{2}\right]^{n-2}-\frac{Q^{2}}{2 r_{+}^{D-2}} & \text { for } D=2 n-1 .\end{cases}
$$

Substituting (4.21) and (4.20) into (3.20), we find the entropy

$$
\begin{aligned}
S & =2 \pi(n-1) \int_{0}^{r_{+}} r_{+}\left[k+\left(\frac{r_{+}}{l}\right)^{2}\right]^{n-2} d r_{+} \\
& =\pi l^{2}\left\{\left[k+\left(\frac{r_{+}}{l}\right)^{2}\right]^{n-1}-k\right\},
\end{aligned}
$$

for the even dimensional black holes, and

$$
\begin{aligned}
S & =4 \pi(n-1) \int_{0}^{r_{+}}\left[k+\left(\frac{r_{+}}{l}\right)^{2}\right]^{n-2} d r_{+} \\
& =4 \pi(n-1) l \sum_{m=0}^{n-2}\left(\begin{array}{c}
n-2 \\
m
\end{array}\right) \frac{1}{2 m+1}\left(\frac{r_{+}}{l}\right)^{2 m+1} k^{n-2-m},
\end{aligned}
$$

for the odd dimensional black holes. When $k=1$, the entropies (4.22) and (4.23) are totally the same as those derived through the Hamiltonian analysis [26]. The method used here seems to be simpler. Furthermore, it is found that the entropy formulas (4.22) and (4.23) are also exactly same as (3.21) and (3.22), which are derived as the charges are absent. It verifies in some sense that the black hole entropy comes from a surface term of gravitational action at the horizon [24, 31, 32]. That is, the black hole entropy is not explicitly related to the Lagrangian of matters.

Finally, we give the heat capacity of charged black holes

$$
C_{Q}=\frac{2 \pi(n-1) r_{+}^{2}\left[\triangle^{n-2}\left[(2 n-1)\left(\frac{r_{+}}{l}\right)^{2}+k\right]-\frac{Q^{2}}{r_{+}^{D-2}}\right]}{\left[(2 n-1)\left(\frac{r_{+}}{l}\right)^{2}-k\right]+\frac{Q^{2} \triangle^{1-n}}{r_{+}^{D-2}}\left[(2 n-1) k+(4 n-5)\left(\frac{r_{+}}{l}\right)^{2}\right]}
$$

in the even dimensions;

$$
C_{Q}=\frac{4 \pi(n-1) r_{+}\left[\frac{4}{l^{2}} \triangle^{n-2}-\frac{Q^{2}}{(n-1) r_{+}^{D-1}}\right]}{\frac{4}{l^{2}}+\frac{Q^{2} \triangle^{1-n}}{(n-1) r_{+}^{D-1}}\left[(2 n-3) k+(4 n-7)\left(\frac{r_{+}}{l}\right)^{2}\right]}
$$


in the odd dimensions, where $\Delta=k+\left(r_{+} / l\right)^{2}$. The behavior of heat capacity is quite interesting. Inspecting (4.25), for the odd dimensional black holes, one can see that $C_{Q}$ is always positive and finite. And $C_{Q}=0$ when $4 \triangle^{n-2} / l^{2}=Q^{2} /(n-1) r_{+}^{D-1}$, which corresponds to the extremal black holes, thereby the Hawking temperature (4.20) vanishes. For the even dimensional black holes, when $k=1$, the heat capacity has been analyzed in [36]. The heat capacity may be positive and negative, between them the heat capacity has an infinite discontinuity. In the physical parameter regime, there are three possibilities: the heat capacity has two, one and no infinite discontinuity(ies). When $k=0$ and $k=-1$, we find that the heat capacity (4.24) is always positive and finite. When $T=0, C_{Q}=0$. This is the case of extremal black holes.

\section{CONCLUSIONS}

In this work we have investigated the topological black holes in the dimensionally continued gravity which is a special class of the Lovelock gravity. This is achieved by embedding the Lorentz group $S O(D-1,1)$ into the anti-de Sitter group $S O(D-1,2)$. In this way the Lovelock gravity is divided into two branches depending on the spacetime dimension: even and odd dimensional cases. The action is, in the odd dimensions, the Chern-Simons form for the anti-de Sitter group and, in the even dimensions, the Euler density constructed with the Lorentz part of the anti-de Sitter curvature tensor. In the cases $D=3$ and $D=4$, the two actions reduce to the Einstein-Hilbert action with a negative cosmological constant in the general relativity. The Lovelock coefficients are reduced to two parameters: cosmological constant and gravitational constant. The horizons of these topological black holes are $(D-2)$-dimensional hypersurfaces with constant positive, zero or negative curvature $(D-2) k$. Therefore, the even horizon of black holes is no longer the $(D-2)$-dimensional sphere $S^{D-2}$. The horizons may also be toroidal or higher genus Riemann surfaces.

We have studied the three kinds of black holes and discussed their thermodynamic properties. From the first law of thermodynamics of black holes, we have calculated these topological black hole entropy. It turns out that the entropy does not obey the usual area formula. When $k=1$, it reduces to that derived through a Hamiltonian analysis for the spherically symmetric black holes [26]. Due to the different topological structures, these black holes manifest different thermodynamic behaviors. In the case $k=1$, the vacuum state is the anti-de Sitter space in the even dimensions and in the odd dimensions, is zero mass black hole solution, as happens in the BTZ solution [1, 33], for which the horizon and singularity coincide with each other at $r=0$. The heat capacity is alway positive for the odd dimensional black holes, but for the even dimensional black holes, positive as $r_{+}>l / \sqrt{2 n-1}$ and negative as $r_{+}<l / \sqrt{2 n-1}$. That is, it has a transition point at $r_{+}=l / \sqrt{2 n-1}$, thereby the heat capacity suffers from an infinite discontinuity. In the case $k=0$, the vacuum state for both solutions is the zero mass black hole as the zero mass BTZ solution. In this case, the heat capacity is always positive for both solutions. In the case $k=-1$, the vacuum state describes a black hole with horizon $r_{+}=l$ for both solutions. This vacuum solution has some peculiar properties. For the different dimensions, the Hawking temperature is same as $T=1 / 2 \pi l$, but the entropy has different behaviors in the even dimensions and odd dimensions (3.45). This is because embedded in the different gravity, the same black hole solution may have different entropy (Note that the vacuum solution in (11.3) when $k=-1$ 
and $M=Q=0$, has a entropy proportional to its area). In this case, the heat capacity is also always positive for arbitrary dimensional black holes. In addition, the negative mass spectrum is allowed in the black hole solutions when $n=2 \tilde{k}+1$, where $\tilde{k} \in \mathcal{Z}$. But, there exists a critical negative mass, beyond which the singularity at $r=0$ will be naked. The critical mass has been found. Recently, the higher dimensional Chern-Simons supergarvity has been investigated [37,38. It would be interesting to study the supersymmetry of these topological black hole solutions found in this paper and the constant curvature black holes [1]

The charged topological black holes in this dimensionally continued gravity have been also considered. The Hawking temperature, entropy and heat capacity have been calculated and analyzed. It has been found that for $k=0$ and $k=-1$ black hole solutions (including those solutions (1.3) in the Einstein-Maxwell theory see the Appendix), the heat capacity is always positive, which means these black holes are more stable than the $k=1$ black holes. This work has extended the investigation on the static, spherically symmetric black holes in the dimensionally continued gravity [26].

\section{ACKNOWLEDGMENTS}

This work was supported by the Center for Theoretical Physics of Seoul National University.

\section{APPENDIX: THERMODYNAMICS OF HIGHER DIMENSIONAL TOPOLOGICAL BLACK HOLES IN THE EINSTEIN-MAXWELL THEORY}

In this appendix, we briefly discuss thermodynamics of the higher dimensional topological black holes (1.3) in the Einstein-Maxwell theory with a negative cosmological constant. For the discussion in the four dimensions see [11]. For the solution (1.3), the vacuum state is

$$
d s^{2}=-\left[k+(r / l)^{2}\right] d t^{2}+\left[k+(r / l)^{2}\right]^{-1} d r^{2}+r^{2} d \Sigma_{D-2}^{2},
$$

which is a $D$-dimensional anti-de Sitter space with a $(D-2)$-dimensional hypersurface $\Sigma_{D-2}$ whose curvature is a constant $(D-2) k$. Therefore, asymptotically, the solution (1.3) is also locally isometric to the anti-de Sitter space. For the vacuum solution (A1), as discussed in the text, the horizon is absent as $k=1$, at $r_{+}=0$ coinciding with the singularity at $r=0$ when $k=0$, and is $r_{+}=l$ when $k=-1$. Differing from the zero mass black hole (3.43) in the dimensionally continued gravity, the zero mass black hole (A1) in the Einstein gravity obeys the area formula of entropy, which we will prove.

The solutions (1.3) may have two, one and no horizon(s). When the solutions describe black holes with non-degenerate horizon, using (3.16), we get the Hawking temperature in terms of the charge and horizon radius $r_{+}$,

$$
T=\frac{1}{4 \pi r_{+}}\left[(D-3) k+(D-1)\left(\frac{r_{+}}{l}\right)^{2}-\frac{16 \pi^{2}(D-3) Q^{2}}{\omega_{D-2}^{2} r_{+}^{2(D-3)}}\right]
$$

From the definition of horizon $g_{t t}\left(r_{+}\right)=0$, we have 


$$
\left(\frac{\partial M}{\partial r_{+}}\right)_{Q}=\frac{(D-2) \omega_{D-2} r_{+}^{D-4}}{16 \pi}\left[(D-3) k+(D-1)\left(\frac{r_{+}}{l}\right)^{2}-\frac{16 \pi^{2}(D-3) Q^{2}}{\omega_{D-2}^{2} r_{+}^{2(D-3)}}\right]
$$

Substituting ( $\mathrm{A2})$ and ( $\mathrm{A3})$ in to $(\overline{3.20})$, one has

$$
\begin{aligned}
S & =\int_{0}^{r_{+}} T^{-1}\left(\frac{\partial M}{\partial r_{+}}\right)_{Q} d r_{+} \\
& =\frac{(D-2) \omega_{D-2}}{4} \int_{0}^{r_{+}} r_{+}^{D-3} d r_{+} \\
& =\frac{1}{4} \omega_{D-2} r_{+}^{D-2}
\end{aligned}
$$

which is just one quarter of the horizon area. It also verifies that the black hole entropy in the Einstein gravity always satisfies the usual area formula, independent of the topology of event horizon. One may wonder whether the entropy of $k=-1$ zero mass black holes (3.43) and (A1) obeys the entropy formula derived from (3.20), because the formula (3.20) seems non-applicable to these zero mass black holes. Recall that the black hole entropy in fact comes from a surface term of the gravitational action under consideration, which is computed at the black hole horizon $r_{+}$[24, 31, 32]. Therefore, the black hole entropy is not related to whether the mass of black holes is zero or not. And hence the result from (3.20) is applicable to the zero mass black holes.

The heat capacity of the black holes (1.3) is

$$
C_{Q}=\frac{(D-2) \omega_{D-2} r_{+}^{D-2}}{4} \frac{\left[(D-3) k+(D-1)\left(\frac{r_{+}}{l}\right)^{2}-\frac{16 \pi^{2}(D-3) Q^{2}}{\omega_{D-2}^{2} r_{+}^{2(D-3)}}\right]}{\left[-(D-3) k+3(D-1)\left(\frac{r_{+}}{l}\right)^{2}+\frac{16 \pi^{2}(D-3)(2 D-5) Q^{2}}{\omega_{D-2}^{2} r_{+}^{2(D-3)}}\right]}
$$

Inspecting the heat capacity, obviously, when $k=0$ and $k=-1$, one may find that it is always positive and finite. When $k=1$, it may be positive and negative, between them an infinite discontinuity occurs, which happens as the denominator of (A5) vanishes. When the two horizons of black holes coincide with each other, we have $T=C_{Q}=0$, which corresponds to the extremal black holes.

When $k=-1$, there is also the negative mass black holes. The critical mass, beyond which the singularity at $r=0$ will be naked, is

$$
M_{\mathrm{c}}=-\frac{(D-2) \omega_{D-2} r_{c}^{D-3}}{8 \pi}\left[1-\frac{D-2}{D-3}\left(\frac{r_{c}}{l}\right)^{2}\right]
$$

where $r_{c}$ satisfies

$$
r_{c}=l \sqrt{\frac{D-3}{D-1}}\left[1+\frac{16 \pi^{2} Q^{2}}{\omega_{D-2}^{2} r_{c}^{2(D-3)}}\right]^{1 / 2}
$$




\section{REFERENCES}

[1] M. Bañados, C. Teitelboim, and J. Zanelli, Phys. Rev. Lett. 69, 1849 (1992).

[2] M. Bañados, M. Henneaux, C. Teitelboim, and J. Zanelli, Phys. Rev. D 48, 1506 (1993).

[3] S. Aminneborg, I. Bengtsson, S. Holst, and P. Peldan, Class. Quantum Grav. 132707 (1996).

[4] M. Bañados, Phys. Rev. D 571068 (1998); M. Bañados, A. Gomberoff, and C. Martínez, hep-th/9805087.

[5] J. D. E. Creighton and R. B. Mann, Phys. Rev. D 58, 024013 (1998).

[6] J. P. S. Lemos, Phys. Lett. B 353, 46 (1995); Class. Quantum Grav. 12, 1081 (1995); J. P. S. Lemos and V. T. Zanchin, Phys. Rev. D 543840 (1996).

[7] C. G. Huang and C. B. Liang, Phys. Lett. A 201, 27 (1995); C. G. Huang, Acta Phys. Sin. 4, 617 (1996).

[8] R. G. Cai and Y. Z. Zhang, Phys. Rev. D 54, 4891 (1996).

[9] D. Klemm, V. Moretti, and L. Vanzo, Phys. Rev. D 57, 6127 (1998).

[10] D. Klemm, gr-qc/9808051.

[11] D. R. Brill, J. Louko, and P. Peldan, Phys. Rev. D 56, 3600 (1997).

[12] L. Vanzo, Phys. Rev. D 56, 6475 (1997).

[13] R. B. Mann, Class. Quantum Grav. 14, L109 (1997); Nucl. Phys. B516, 357 (1998).

[14] R. B. Mann, Class. Quantum Grav. 14, 2927 (1997); W. L. Smith and R. B. Mann, Phys. Rev. D 56, 4942 (1997).

[15] J. P. S. Lemos, Phys. Rev. D 57, 4600 (1998).

[16] R. G. Cai, Nucl. Phys. B 524, 639 (1998).

[17] D. Birmingham, Phys. Lett. B 428, 263 (1998).

[18] M. M. Caldarelli, gr-qc/9802024.

[19] D. Klemm and L. Vanzo, gr-qc/9803061.

[20] A. DeBenedictis, gr-qc/9804032.

[21] M. M. Caldarelli and D. Klemm, hep-th/9808097.

[22] R. G. Cai, J. Y. Ji, and K. S. Soh, Phys. Rev. D 57, 6547 (1998).

[23] R. C. Myers and M. J. Perry, Ann. Phys. (N.Y.) 172, 304 (1986)

[24] R. G. Cai and Y. S. Myung, Phys. Rev. D 56, 3466 (1997).

[25] D. Birmingham, hep-th/9808032.

[26] M. Bañados, C. Teitelboim, and J. Zanelli, Phys. Rev. D 49, 975 (1994).

[27] D. Lovelock, J. Math. Phys.(N.Y.) 12, 498 (1971).

[28] A. Ilha and J. P. S. Lemos, Phys. Rev. D 55, 1788 (1997).

[29] X. Z. Li, Phys. Rev. D 50, 3787 (1994).

[30] C. Teitelboim and J. Zanelli, Class. Quantum Grav. 4, L125 (1987).

[31] T. Jacobson and R. Myers, Phys. Rev. Lett. 70, 3684 (1993).

[32] M. Bañados, C. Teitelboim, and J. Zanelli, Phys. Rev. Lett. 72, 957 (1994).

[33] O. Coussaert and M. Henneaux, Phys. Rev. Lett. 72, 183 (1994).

[34] A. Strominger, J. High Energy Phys. 02, 009 (1998).

[35] S. Carlip, J. Gegenberg, and R. B. Mann, Phys. Rev. D 51, 6854 (1995).

[36] J. P. Muniain and D. Píriz, Phys. Rev. D 53, 816 (1996).

[37] R. Troncoso and J. Zanelli, hep-th/9710180.

[38] M. Bañados, gr-qc/9803002. 